The universal curves can be used for multilayer earths to the same degree of approximation as the methods discussed by Hummel and Tagg, and, in fact, this approximation can be rapidly and easily accomplished.

This method has been used on four experimental curves obtained by L. S. Palmer. In one case, bore. hole data were available and the result was in ex. cellent agreement for the depth of the layer.

J. M. Hough

Physics Department,

University College, Hull.

Jan. 30 .

${ }^{1}$ Wenner, F., U.S. Bur. Stand. Sci. Paper, 258, 469 (1917).

2 Hummel, J. W., Amer. Inst. Min. and Met. Eng., Tech. Paper No. 418

${ }^{3}$ Tagg, G. F., Amer. Inst. Min. and Met. Eng., Tech. Paper No. 755.

- Roman, I., U.S. Dept. of Commerce, Bur. of Mines, Tech. Paper No. 502 .

\section{Annealing of Glass}

S. M. Cox $^{1}$ has considered the departure from the 'Maxwellian' law of the annealing-rate in glass. He attributes that part of the discrepancy which cannot be accounted for on the basis of a progressively changing viscosity to changes in the equilibrium condition of the glass under the action of the stresses present. An expression for the rate of release of stress is obtained, based on a theoretical derivation of the laws governing these changes and the rate of approach to equilibrium. Unfortunately, in his brief account only the final expression is given, so that it is not possible to comment on the derivation, nor on the extent to which a fit with Morey's experimental results provides a confirmation of the theory.

However, these approaches to an equilibrium condition in the presence of stresses are well known as observed phenomena, appearing as the 'delayed elastic effects' in ordinary experiments. Under constant stress, glass and many other substances obey the following expression :

$$
\text { strain }=a+b t+c \cdot f(t),
$$

where $a, b, c$ are constants proportional to the stress and dependent upon the temperature, composition, etc. The three terms represent respectively the instantaneous reversible elastic strain, the irreversible purely viscous flow at constant rate, and the reversible delayed elastic strain. The function $c . f(t)$ has the value 0 at $t=0$ and $c$ at $t=\infty$.

It is scarcely surprising that the rate of release of stress during annealing is not 'Maxwellian', when it is remembered that Maxwell's equation of visco. elasticity (which suggests the 'Maxwellian' or exponential rate of release of stress) leads to an expression for the behaviour under constant stress which does not include any delayed elastic effect, namely : strain $=a+b t$. The original theory of annealing, in fact, considered only relaxation of stress through the mechanism of viscous flow. The representation of the more complicated behaviour of real materials has, however, been extensively discussed (see, for example, Alfrey and Doty ${ }^{2}$ ), and it is possible to derive a theoretical 'stress release' curve from any 'creep' curve obtained under constant applied stress, and vice versa.

I have recently shown ${ }^{3}$, by considering the mech anism of the annealing process and the experimental data on the delayed elastic effects in glass, that it would be possible on this basis to account for the discrepancies remaining in the theory of annealing. I pointed out also that a further complication existed, since the rate of release of stress must depend partly on the form of the previous cooling schedule for the glass, during which partially developed delayed elastic strains might have been 'frozen-in'. Experimental data in this field are at present insufficient for a quantitative formulation of the annealing laws.

The description of the phenomena proposed by Mr. Cox appears to be analogous to the explanation of delayed elasticity under uniform compression described by Frenkel ${ }^{4}$. It is of interest that this explanation, and apparently Mr. Cox's theory, both lead to a single exponential term of the form $\{1-\exp (-t / \tau)\}$ for $f(t)$, although the experimental results on the ordinary delayed elastic effects can only be represented as the sum of a number of such terms with differing coefficients and 'relaxation times'. For glass the function is probably of the form $\{1-$ $\exp (-\sqrt{t / \tau})\}$, which implies a continuous distribution of such terms. There are other discrepancies between this theory and the experimental facts, and added complications in considering stresses, such as shear stresses, which do not involve a change in volume. It is more satisfactory to look to the existing (although incomplete) experimental data for an explanation of the anomalies in the annealing process.

\section{Clarendon Laboratory, Oxford. \\ March 16.}

${ }^{1}$ Cox, S. M., Nature, 161, 401 (1948).

2 Alfrey, T., and Doty, P., J. Appl. Phys., 16, 700 (1945).

${ }^{3}$ Jones, G. O., J. Soc. Glass Tech., 31, 218 (1947).

"Frenkel, J., "Kinetic Theory of Liquids" (Oxford, 1946).

Ir is true that such theoretical work on problems associated with annealing of glass must be of a tentative nature until adequately supported by experiments, but its value lies in that it provides guidance for the design and interpretation of experiments. Without such guidance there is, for example, confusion between delayed elasticity and viscosity change with time; the influence of stabilization with respect to temperature is also enigmatical. In Dr. Jones's own experiments ${ }^{1}$ no attempt was made to stabilize the glass with respect to temperature. His results are particularly interesting for this very reason, because in his bending test-involving an averaged effect for both tension and compression together-there are theoretical grounds for supposing concurrent temperature stabilization to be without influence. Dr. Jones, in finding this experimentally, erroneously concludes the effect to be absent.

A feature which reduces the usefulness of the experimental results is that glasses of different composition have been used, and unfortunately there is a sparsity of experimental work on 'dilute' silica glasses - the study of which is most likely to be amenable to theoretical treatment.

Research Laboratories,

S. M. $\operatorname{Cox}$

J. A. Jobling and Co., Ltd., Wear Glass Works, Sunderland.

'Jones, G. Q., J. Soc. Glass Tech., 28, 432 (1944). 